\title{
Poblik Prokyorment Between dih Karibyan Kontry Dem ahn dih Archipelago a San Andres, Pravidens ahn Kethlena
}

\section{Hillary Yohanna Rodríguez Mártinez ${ }^{2}$}

Language: Kriol English. (San Andres, Providence and Kethlena official language).

\section{ABSTRAK}

Dih aydia a disa artikl dah fih bring up dih apartunity bejain dih CARICOM fih dih archipelago a San Andres, Pravidens ahn Kethlena ina dih sens fih poblik kantrak. Deh embries dih fac seh wih shier history, koltyo ahn ivn economical fiitiors wid dem kontry weh dah memba. CARICOM still gah tings fih beta, bot definitely ih gud fih staat consida ih seriously becaaz dis absoluutly gwain bii a giem chienja fih dih economy a dih karibyan, espeshally fih poblik entity dem. Forss ih taak bout dih dih Chaguarama triity ahn den dih chalenj dem fih poblik kantrak ina dih karibyan ahn how ih kya work fih wih archipelago.

1 Spelling ahn gramma rivaisment by Professor Christopher Adel Livingston.

2 Lawyer from the San Buenaventura University, Cali, Colombia. Master's degree in Law and Economycs from the Universidad Externado de Colombia, Bogotá, Colombia. Public employee at the Governance of the Archipelago of San Andres, Providence and Kethlena, San Andrés, Colombia. Correo-e: gilaryromar@outlook.com. Enlace ORCID: https://orcid org/0000-0002-4272-3376. Fecha de recepción: 11 de abril de 2020. Fecha de modificación: agosto 2 de 2020. Fecha de aceptación: 20 de septiembre de 2020. For citation of this article: Rodríguez Martínez, Hillary Yohanna, "Poblik Prokyorment Between dih Karibyan Kontry Dem ahn dih Archipelago a San Andres, Pravidens ahn Kethlena", Revista digital de Derecho Administrativo, Universidad Externado de Colombia, n. ${ }^{\circ} 25,2021$, pp 429-441. DOI: https://doi.org/10.18601/21452946.n25.14. 
Kiiwords: karibyan, economi, San Andres Aylant, CARICOM, poblik kantrak, internashonal kaaperieshan.

\section{Contratación pública entre el Caribe y el archipiélago de San Andrés, Providencia y Santa Catalina}

\section{RESUMEN}

El presente artículo busca resaltar la oportunidad que puede representar al archipiélago de San Andrés, Providencia y Santa Catalina la comunidad del Caribe CARICOM, aprovechando patrones históricos, culturales y económicos comunes con los países miembros y que pueden facilitar el acercamiento con estos. Si bien el CARICOM aún tiene un largo camino por recorrer, es importante empezar a considerarlo como un dinamizador de juego en la economía del Caribe, en especial manera, para entidades públicas. En primera medida se revisa el Tratado de Chaguaramas y, por último, los desafíos que enfrenta la contratación pública en el Caribe y lo que esto significa al archipiélago.

Palabras clave: economía, Caribe, San Andrés isla, CARICOM, contratación estatal, cooperación internacional.

\section{Public Procurement Between the Caribbean Countries and the Archipelago of San Andres, Providence and Saint Kethlena}

\section{ABSTRACT}

This paper aims to highlight the opportunity presented by CARICOM for the Archipelago of San Andres, Providence and Kethlena in Public Procurement, leveraging the common cultural, historical and economycal features with the member states that facilitate the approchement between the Archipelago and the member countries of CARICOM. Though CARICOM still have a long way to go, it is important to begin to consider it as a game changer for the Caribbean economy, as it can also be for our islands and, especially, for its public entities. This work first examines the Chaguaramas Treaty and ends with the challenges that Public Procurement tackle in the Caribbean region and what it means for the Archipelago.

Keywords: Caribbean, Economy, San Andres Island, CARICOM, Public Procurement, International Cooperation. 


\section{IINTRADOKSHAN}

San Andres, Pravidens and Kethlena dah wan archipelago weh lokiet $775 \mathrm{~km}$ fahn Colombia, $400 \mathrm{~km}$ fahn Jamaica and $220 \mathrm{~km}$ fahn Nicaragua Atlantic cuost. Truu dis posishan Colombia gat baada lain wid Panamá, Costa Rica, Nicaragua, Honduras, Jamaica, Haiti ahn Dominican Republic ${ }^{3}$. Fahn taim to taim demia ayslant dem caal atenshan fih gud bot siem wie fih bad, becaaz ih gah wan ubikieshan weh mek dih mobility ina dih Karibyan, to naat ahn central America, iisia. Ina old taims dah wehn dih pairat dem ahn nowadies dih drog diila dem$^{4}$. Dih thing dah seh wid aal seh wih gat dem pail a baada lain, luck laik dih iliigal activity dem deh embries it moe dan dih legal wan dem $_{i}$ ina poblik matta for example, dih poblik Entity dem fahn dih archipelago only mek commercial bisnis wid dih siem compatriots dem uovayanda. Ivn afta dem mek dih law 915 fahn $2004^{[5]}$.

Dah no intenshan a disia artikl fih taak bout dih efichensy a dih poblik kantrak dem San Andres, bot still, wih haffi bring op dih fac a seh fih wih productivity luo and wih biely gat local indostry, so dih fiw aylandaz dem weh sell tu dih poblik entity dem iinia end up deh mek suoso intermedieshan, ahn all dat du dah mek tings diera fih dih govament ahn fi wihself, becaaz ih kiip back dih entity dem fah get benefit dem supuos to get afa dih maakit, laik beta valio fih prais.

Miinwail, wih karibyan nieba dem don andastan dih ruol weh dih globalizieshan deh plie ina dih divelopment a dih difarent kontry dem, so weh dem do dah get togeda ahn organaiz demself as CARICOM (1973) weh forst when niem CARIFTA $^{6}$. Dih aydia a dih CARICOM presaisly dah fih dih kontry dem weh dah memba join demself ina wan single maakit and cooperiet wid wan anada fih muuv roun dih difarent merchandaiz weh dem prodius. Dem dah no wan political nar monetary organizieshan but prabbly dem wih suun get tu daty

3 Jesús SALAS, Reflexiones socio-bistóricas sobre el turismo en el Caribe insular colombiano: una lectura del Puerto Libre, Tesis de grado, Universidad Nacional, Bogotá, pp. 21-22.

4 Andrés SÁnChez JabBA, "Violencia y narcotráfico en San Andrés", Documentos de trabajo sobre economía regional n.181, Banco de la República, febrero de 2013. Disponible en línea: https:// www.banrep.gov.co/sites/default/files/publicaciones/archivos/dtser_181.pdf [consultado el $1 .{ }^{\circ}$ de agosto de 2020].

5 Ley 915 de 2004, artículos 2 y ss. Gaceta Oficial, 21 de octubre de 2004.

6 The establishment of the Caribbean Community and Common Market (CARICOM) was the result of a 15-year effort to fulfil the hope of regional integration which was born with the establishment of the British West Indies Federation in 1958. The West Indies Federation came to an end in 1962 but its end, may be regarded as the real beginning of what is now the Caribbean Community. Caribiean Community Secretariat (Caricom), "History of Caribbean community, Guyana". Available online: https://caricom.org/historyof-the-caribbean-community/\#: :text=The\%20Caribbean\%20Community\%20and\%20 Common\%20Market\%20(CARICOM)\%20was\%20established\%20by, effect\%20on\%20August $\% 201 \% 2$ C\%201973 [consulted 03/03/2020]. 
paint. Dih gud niws dah seh fih wih aylant dem shier historical ahn cultural backgrong wid dem kontry ahn wih economy bies afa dih touristic activity in forst plies just laik dem, ivn seh wih dah no CARICOM memba.

Laik allmost all dih rest a singl maakit triity dem, CARICOM gah wan protocuol fih poblik kantrak, so, wan a dih paint wih haffi go uova dah, wat if Colombia cud embries dih speshal kandishans a dih archipelago fih siik faward wan integrieshan tu dih triity weh cuda pormit dih archipelago fih get dih moch a dih poblik kantrak protocuol? Dis kuda bring benefit fih wih kansidaring seh wih kuda bay fahn pliesis niera tu wih, ahn siem wie dih aylandaz dem weh gat bisnis can sell tu dembe kontry tu, so wih activiet a comershal muuvment bitwin dih CARICOM memba dem ahn wii. Well In fac, Ina 1994 Colombia saing op tuu triitis rilietid tu dih CARICOM, wan wos dih agriiment bitwin dih Karibyan komiunity (CARICOM) ahn dih govament a Colombia pahn tried, economyc ahn technical cooperieshan and (1994), dih ada wan dah dih agriiment weh kanstityut dih asocieshan a dih karibyan stiets dem weh soscraib dih 24 a Julai 1994, ah dehn get ratifai bai dih laa 216 fah 1995. Onfortunietly, non a dem neva divelop muo afta dem get saign.

CARICOM still gah tings fih beta, bot definietly ih gud fih Colombia staat consida it seriously becaaz dis kya signifai a giem chienja fih dih economy a dih ayslant, espeshally fih poblik entity dem, since before dih COVID-19 dih karibyan contry dem wehn deh experience a riil pasitive growth ${ }^{7}$. Dih Cepal present wan gruo projecshan fih 2019 pahn 1.4, ahn 5.6 fih 2020 ina dih riijan ${ }^{8}$ Colombia insular riijan gat som guud paint pahn im fieva, laik dih karibyan aidentity, dih lokieshan, and dih kandishan a frii puort, for exampl. In aada fih kya andastan dih actual situeshan a dih archipielago towards dih CARICOM, disia article gwain go uova dih fallowing tapics I) dih chaguaramas rivais triiti II) How dih poblik prokyorment iina dih karibyan deh work afta dih chaguaramas rivais triiti ahn III) ih gwain finish wid som kanclushans.

\section{DIH CHAGUARAMAS RIVAIS TRIITI}

Sins 1973 dih karibyan kontry dem get tugeda ahn kanfarm dih CARICOM -Caribbean Community, well knuon tu as dih revais triity fah Chaguaramas. Dih memba dem fih tude dah 20, Antigua ahn Barbuda, Bahamas, Barbados, Belize, Dominica, Granada, Guyana, Haiti, Jamaica, Montserrat, Saint Lucía, St Kitts

7 Interamerican Development Bank (IDB), "Caribbean region quarterly bulletin, A look at 2019 and beyond", vol. 8, n. ${ }^{\circ}$ 4, 2019. Available online: https://publications.iadb.org/ publications/english/document/Caribbean_Region_Quarterly_Bulletin_Volume_8_Issue_4_December_2019_en.pdf [consulted 03/07/2020].

8 CePAL, "Latin America and the Caribbean: Growth Projections for 2019 and 2020, 2019". Available online: https://www.cepal.org/sites/default/files/pr/files/table_press_gdp_preliminaryoverview2019-eng.pdf [consulted 03/07/2020]. 
ahn Nevis, St Vincent ahnGranadines, Suriname ahn Trinidad \& Tobago. Yo gat som kontry weh asoshiet, demya dah Anguila, Bermuda, British virgins aylant, Cayman aylant, ahn Turks ahn Kaycos aylant. ${ }^{9}$ Iivn seh demia kontry wan bay wan no gat strent, if dem work unait dem kya gat som kain a influens ina dih internashonal friem ${ }^{10}$.

Dih aydia a dih CARICOM prisaisly dah seh, bies pahn dih similar background fah aaal demya kontry, dem kyan work tugeda fih wan economyc development iina paadnaship. Dih triity inkluud dih commitment a dih Stiets dem fih mek wan protocuol bout poblik prokyorment ${ }^{11}$, bikaaz dem rialaiz wan big fak weh dah dih waid economy weh kyan muuv chruu dih poblik kantrak dem, ahn seh work separietly kyan kiip bak wan aylant fahn get dih tings dem ihn niid. Imajin for exampl how yuusful ih kyan biii ina dih migl a disaasta laik wan harykien fih demya aylant kyan help wan anada tray get dih tings dem weh dem niid fih get uova dem difikolty ahn no haffi wiet till ada mienlan seni fih dem. Dih protokuol bies pahn di conshosnes a seh dih memba dem kya sekior demself dih best valio fih dem mony as lang as dem kya put in practis prinsipl laik transparensy, ikwal triitment ahn kompetens ${ }^{12}$.

Dizya protokuol pik op dih rekomendieshan ah Schrouder (2010) weh propuos fih dih karybuan kontry dem insentiviet dih kompetens, sentralais dih pruoses dem, Impruuv teknolojy ahn fuokos pahn produktovity (pp. 8-10 $)^{13}$. Indiid, demya dieez iina dih web piej fih CARICOM yoh kyan fain dih prokyorment nuotis dem, weh dah wan instrument weh dih kontry dem weh don adap dih protocuol deh yuuz fih mek poblik dih niids weh dem waah kantrak, so eny soplaya fram eny wan a dih kontry dem weh dah memba, kyan prizent a bid ahn get dih kantrak.

Dih laa 915 fahn 2004, Colombia ${ }^{14}$, limit dih partisipieshan or beta seh ih gih a prefrensy tuh dih aylandaz dem fahn San Andres, fih sel dih govament

9 Caribbean Community (CARICOM), "Member states and associate members, 2020". Available online: https://caricom.org/member-states-and-associate-members/ [consulted 03/03/2020].

10 Christopher P. Malcolm, "Caribbean Integration Within the Caricom Framework: The Socio-Historical, Economyc, And Political Dynamics of a Regional Response to A Global Phenomenon", Law \& Business Review of the Americas, SMu, Dedman School of Law, 2004 pp. $11-13$.

11 Article 239 reads as fallow: "The Member States undertake to elaborate a Protocol relating, inter alia, to: (a) electronic commerce; $($ b) government procurement (c) treatment of goods produced in free zones and similar jurisdictions $;$ (d) free circulation of goods in the CSME, and (e) rights contingent on establishment, provision of services and movement of capital in the Community".

12 Caribbean Community (Caricom), Draft Protocol on Public Procurement for The Caribbean Community, Guyana: Gerogeown, 2017, pp. 7-8.

13 Sandra Schrouder, Public Procurement in The Caribbean: Confronting the Challenges and Opportunities, Florida: Miami Shores, Barry University, 2010, pp. 8-10.

14 Article 67 a dih laa 915 fah 2004 riid as: "kantrak: ina licitieshan ahn kantraks weh fih exekyut ina dih archipelago department, dih entity weh deh licitiet fih luck ata wan riil 
wehn dih kantrac fih divelop ina dih archipelago. Dah no bad atall taakin bout ekwity, bicaaz dah wan gud instrument fih divelop fih wii internal economy, bot if wii wuda waa workout som kain a agreement wid dih CARICOM, prabbly dis wuda haffi pass chruu som modifikieshan, sins wan a dih principle ina dem kain a triitiz dah dih iqual triitment fih dih citizens dem fahn dih kontry dem weh saign op. Bot enywie, dih fak dah seh iivn wid dis mejament dih prodoktivity iina dih aylant no deh gruo, tudeh kantriery, ih luk laik die afta die tuorism deh akapariet aal dih han work a dih aylant; wat hapm dah seh stil aylandaz bay fram dih intiria ahn sel uova tuh dih govament, wih prodokshan is luo ahn dat kiip wih prais dem deh go op ${ }^{15}$.

Ina 1994 Colombia saign tuu agriiment wid dih karibyan comiunity. One was dih "agriiment bitwiin dih karibyan comiunity ahn dih govament a Colombia pahn tried, ekonomyc ahn technical coperieshan" -agreement between the caribbean community (CARICOM) and the government of the Republic of Colombia on trade, economic and technical co-operation (1994)- ahn dih nada wan dah dih agreement weh kanstityut dih karibyan stiets asocieshan. Dih tuu a dem get saign dih siem yer Cartagena. Bot as how dih kansila fah Colombia, Carlos Homes Trujillo himsself seh ina dih forst kansila somit, last yer, juun 2019, demya agriiment stie pahn piepa, dem neva get moe divelopment. Akarding to dih niws dem, ina dis miiting dih kansila dem compromais demself fih push ahn an mek efectiv dih cooperieshan bitwiin dem ina several fiil ${ }^{16}$.

If dis go chruu, San Andres ahn dih aylandaz dem kuda get riil benefit afa triitiz laik dih chaguaramas (CARICOM) ahn kriet a biga maakit widd ih nieba dem. Nuotis seh tuu a dih CARICOM memba, Jamaica and Haiti, dem dah jiografikal limit tuh dih aylant; ih uda bii griet if aylandaz kuda sel tu govament ahn poblik entitis from demia aylant ahn at dih siem taim mek dem cuda sell wii. Dis no ongl uda bain bak (tay bak tait) dih koltyoral ahn historical chiens bitwiin din karibyan, bot uda permit dih archipelago fih get betta valio fih mony as wih bay fram fih wih mienlan Colombia ahn also fram fih wih nieba dem, bicaaz dih aktiviet kompetens between Colombyan soplayars ahn karibyan soplayars; ahn kompetens in economy is alwies siims laik benefishal. Plus, as karibyan dih ayslant dem diil wid dih siem problematik, ahn som a demya kontry don laan how fih get uova tings weh wii still no kantruol ahn weh Colombya no gat expiriens or manijment fah, sins dih mienlan topography ahn

participieshan a dih raizal ahn resident dem, deh gih valyo tu disa fact. Ahn ina iqual kandyshan ih gwain rada dih raizal ahn resident dem fah dih Dipartment". tesis de maestría, Universidad Externado de Colombia, Bogotá, 2018, pp. 78-94.

16 Agencia EFE, "Colombia y Caricom acuerdan estrechar lazos y hacer crecer su comercio común". Available online: https://www.efe.com/efe/america/politica/colombia-y-caricomacuerdan-estrechar-lazos-hacer-crecer-su-comercio-comun/20000035-4012286 [consulted 03/07/2020]. 
claimit kandishan plenty difarent fah fih dih ayslant. Nevadiless dih chalenj fih dih aylandas dem gwain bii fih develop mekanism fih mek shuor wih no end up deh droundid dih likl prodokshan wih gat.

Ivn seh tudeh dies CARICOM deh fies ihm uno chalenj dem ${ }^{17}$, kiip in main seh eny agreement weh wan kontry kya do fih incentiviet internashonal cooperieshan weh uopn duo fih ihm uon piipl gwain alwies qualifai as a cluosa step tu him uon divelopment. Dih duo don upon wid CARICOM crhuu dih agriiment dem weh dih kontry don saign, ahn dah just a mata a mek it hapn ahn ahn iivn extend it tu dih poblik prokyorment.

Som mie seh, dih agriiment don de deh ahn ih uopn fih aylandaz embries ih, plos, Colombia guarantii equal triitment tu dih bid dem fahn piipl fah dih kontry dem weh ih ga agriiments wid, iivn ina poblik prokyorment; ahn akarding tu dih agriiments, Colombyans supuos tu riciiv dih siem rigaards. Dih paint is, San Andres, Pravidence ahn Kethlina kyan gat a spechall triitment ina dih agriiment betwiin Colombia ahn CARICOM diu tu ihm kandishans such as papulieshan, ancestrality ahn lokyeshan. Pahn tap, dih archipielago niid a biga effort fahn dih national ahn local gavament fih mek demya agriiment a riality fih aylandaz insida stie as ded words pahn dih piepa.

\section{POBLIK PROKYORMENT IINA DIH KARIBYAN AHN SAN ANDRES, PRAVIDENS AHN KETHLENA}

Poblik prokyorment or poblik kantrak ina San Andres dah somting weh haaly call dih atenshan a pipl, exep fih dih wan dem weh liv afa ih or gat wan nier rilieshan wid ih. Dis chienj tuu yierz abak wen dih fiskalia kom ahn gaan wid som ofishal worka, since dehn iivn dih moe kierless porsn gat somting fih seh bout kantrak. Som piipl wen glad biliiv ih uda don wid karopshan, som bes andastan dis dah no ting bout wan nar tuu, dah wan huol komyunity problem ahn karopshan go biyaan a judishal trayal. Afta dat San Andres tek uova dih atenshan a di huol kontry ahn dah neva chruu dih pritinis a dih biich, nard ih swiitnis a dih rondong.

Wel ih gud fih go uova dih mata, ahn tray fain out wat dah Dih fielyo wih gat iina poblik kantrak ahn how wii supuoz tuh diil wid dem. San Andres dah wan a dih smaalis aylant iina Dih Karibyan ahn iivn duo wih no independent laik dih res a wih nieba dem, wih stiliet shier background ahn chalenj wid dem.

Poblik kantrak dah dih wie govament kyan satisfay dih niids a dih komyunity, so wii kyaah ivn tink bout don wid ih, beta wih fuokos hafy deh pahn mek ih muo efishent ahn fruutful. Somtaims, laik ina dih kies fih COVID-19 dih gud fih bii wan aylant ina dih migl a dih karibyan sii, sins dih kandishan a insularity 
permit dih authority dem fih kiip a beta kantruol a dih going ahn coming ah pipl $_{\text {; }}$ bot ada taim dah wan disadvatish ${ }^{18}$.

Dih aysolieshan a dih karibyan aylant dem jinariet som kayna difikolty fih dih provishan a guds ahn sorvisis. Dih economy a dih karibyan bies pahn activity laik turism, fishin, agrikoltyo (ivnduo ih gwain dong) ahn som a dih aylant dem stil gat wan pail a minin gwain aan ${ }^{19-20}$. Dis mek wih seh dih wiiknis a dih Caribbean dah produktivity; iina San Andres kies wii soplay fram Colombia mien land, Costa Rica, ahn Miami, prinsipaly. Dis partikyularity jinariet enywie wan ekstra kaas fih wii as dih laas kansyuma, chruu trasnportieshan ahn adda implikieshans, ahn dah dih siem fih dih govament.

Taakin bout implayment, akarding to wan article poblish bai Sandra schourder in dih yer 2010, dih bigis implaya iina dih karibyan dah dih govament ivn seh turism dah dih economic activity wid muo influens ${ }^{21}$. Dih writa ius a informieshan fram dih World Bank fih ilustriet, weh kanfarm she far example iina dih kies a Jamaica iina dih yierz 2001-2002 dih 78\% a dem poblik bodjet gaan pahn salary ${ }^{22}$. Fih 2018 iina San Andres govament bodjet dih 37\% dah wen fih invesment, ahn dih siem porsentij fih dih founshan a dih institushan ${ }^{23}$. Ivndou seh wih gat wan biga porsentij weh go pahn investment kompier wid Jamaica wih stil fies wan chalinj fih adkuaya dih guds ahn sorvis weh wii niid fi satisfai dih komyunity nesesity. Dih riison wai so moch moni go pahn salary payment, akardin tu dih siem articl dah bikaaz a dih wie palitiks divelop iina demya plies, weh piipl vuot ahn dih fors ting dem expek fram dih wina dah wan jab.

Wan impuortant ting fih seh dah seh dakyumentieshan bout poblik kantrak iina dih Karibyan dah no plenty, yoh gat likl bit refrens bout ih. Wan a dih fiew academic artikl yuh kia fain dah dih piepa weh a menshan abov fan di yer 2010 "poblik prokyorment iina dih karibyan: kanfronting dih chalenj

18 Emily Wilkinson, "COVID-19: A Lesson in Leadership from The Caribbean", Overseas Development Institute. Available online: https://www.odi.org/blogs/16959-covid-19-lessonleadership-caribbean [consulted 15/07/2020].

19 Portafolio, "Aislado San Andrés pierde 98 millones cada mes". Available online: https://www. portafolio.co/economya/aislado-san-andres-pierde-98-000-millones-cada-mes-542467 [consulted 03/03/2020]. Akarding tu budget manija ina dih Department, San Andrés deh luus montly muo dan 32 tousn US Dala.

20 The World Bank, "The World Bank in The Caribbean, The World Bank Is Swiftly Supporting the Efforts of The Caribbean In Facing National Emergencies Caused by COVID-19". Available online: https://www.worldbank.org/en/country/caribbean/overview [consulted 15/07/2020].

21 Sandra Schrouder, Public Procurement in The Caribbean, óp. cit., pp. 1-14.

22 Ibíd., p. 1.

23 Gobernación del Departamento Archiplélago de San Andrés, Providencia y Santa Catalina, Informe de gestión 2018, 2018. Available online: https://www.sanandres.gov.co/documentos/InfGestion2018/INFORME\%20DE\%20GESTION\%202018.pdf [consulted 03/03/2020]. 
dem ahn dih apartiunity"24. Dis piepa mek wih sih dih prablem dem weh dih karibyan kontry dem wen gat ahn stil gat wid dih poblik kantrak. Dis piepa weh Schrouder wrait staat wid a likl history af Poblik prokyorment ahn den drap iina dih situeshan fih dih karibyan ahn shii aidentifai dih problem weh demya aylant gat, laik so:

1) Wan pail a piipl bitout jab weh uovaluod dih poblik section and weh liid wih tu a shaat a ekwity ahn efishensy; 2) Plenty puornis aal a about, ahn dih wie hou wih selek wih riprisentieshan tuh poblik aafis bies pahn popyula vuot weh ongl liid tuh karopshan. Informieshan obtien fram Transparency Internashonal fih dih yier 2009 shuo a karopshan persepshan index fih Haiti (1.8) nier tuh dih batam a dih list wid Jamaica ahn Dominican Republik weh jos deh pahn tap wid a rieting a 3 ; 3) desentralisieshan iina dih govament iina karibyan economy; 4) Dih Non rifaarm prokyorment system dem $_{i}$ 5) Smaal saiz a dih prokyorment bodjet, weh no mek dih likl aylant dem fih kompiit iina dih big maakit dem, ahn bay dem kyaah kompiit iina big maakit, dem kyaah get dih benefit dem dis kuda gih, fih example iina skiel economy ${ }^{25}$.

In lain widis, dih raita also divelop som sojeshan fih impruuv dih poblik prokyorment ina dih karibyan ahn mek it mue efishent: "i) Wan exekyutiv ajensy fih sentralaiz dih pruoses dem. ii) Fuokos muo pahn prodoktivity. iii) Impruuvment iina dih kalekshan ah informieshan ahn iv) dih Modernisieshan ahn skil opgried"26.

Dih poblik prokyorment ina demya karibyan kontry dem almost work jost laik dih Colombian wan, don Jamaica far example, dih organais anda dih "Public Sector procurement policy" ${ }^{\text {"27 }}$ ahn dih principle dem weh ruul ih dah almost dih siem wan dem laik Colombia. Barbados gah ih won laa fih poblik prokyorment tuh ahn ih work simila to fih wih. Siem hapn tuh dih ada ayslant dem. So a unifikieshan ina disya sens, ligaly dah wat dih protokuol weh dih CARICOM propuos pritend, still wehn yuh sarch dih CARICOM webpage yuh no fain dih amount a prokyorment appartiunity weh deh pah dih ofishal webpiej a dih ayslant dem, ahn prably a gud taim ago pass befuor dih CARICOM poblik prokirment nuotis staat fonkshan how dih supuos.

CARICOM wos criet anda dih perspective a wan join karybian. Ahn iivn afta muo dan twenty yierz sinz Colombia mek agriiment wid karibyan komiunity, San Andres no gat non taip a economic kanecshan wid wih nieba dem. Dih wraita refa opa tap, análisis, "is a good look", ahn if wii wuda waa kampier ih

Sandra Schrouder, Public Procurement in The Caribbean, óp. cit., 2010, p. 11.

Ibíd., p. 10.

Ibíd., p. 14.

Ministry of Finance \& Public Service, "Public Sector procurement policy". Available online: https://mof.gov.jm/procurement/public-sector-procurement-policy.html. [consulted 03/07/2020]. 
wid San Andres ahn Pravidens kies, as Jamaica, Barbados ahn ada kontry dem, Colombia don gat wan ajensy fih poblik prokyorment, ahn fih be sincier ih result riily helpful tu dih entity dem weh deh kantrak guds and sorvis ina dih kontry; dih ajensy pick up tuu a dih recomendieshan weh transcraib opa tap deh, wan dah dih sentralizieshan a dih process dem ahn di ada wan dah dih kalekshan ah informieshan. Nevadiles, disia ajensy dah wan internal instrument weh luck fawad fih transparency moe dan integrieshan wid dih nieba dem ahn op tu tudeh die, fiwi only rilieshan fih poblik kactrak dah wid wi mien lan, Colombia.

Wat wih definitely stie bijain wid as a menshan befuor, dah dih prodoctivity. San Andres ahn Pravidens wans dah wehn ayslants weh ius tu prodius ahn survaiv afa ih won food we him prodius, dih aislant dem ivn iustu export prodocts laik coconut ahn katn, dats wai mek unuh sii seh all uova dih aislant full a cotnut plantations ${ }^{28}$. Bot fan dih moment wii bigin chienj dih fuocos a wih economy wih become a dependant aislant, dih iisy fih biliiv dis only deh hapn San Andres ahn Pravidens, bot afta go uova dih economy a dih rest a karibyan aislant dem yuh rialaiz dah wan kaman denominii fih all, siims laik aislandaz dem fain iisia wie fih exploit dih lan wid turism dan wid dih agrikoltyo or adda praimary activitis. Dih cansequence fih dih smalist wan dem laik wii dah seh in craisis taims laik nowadies wid dih corona vairos wih fain wihself deh dipend pahn dih amount a fuud ahn dih prais dem weh com fram dih intiria. Som piipl beliiv dis dah wan apartiunity fih dih govament sii dih liidaship a tuorism ahn sief dih bisnis dem, Bot fahn anada view, dis only shuo seh tuorism dah wan activity weh yuh just kiant rilie pahn, dih territory most prodius ahn dat dah weh wih wiikness deh, san andresans put don dih machet ahn dih strobl fih pik up pen ahn piepa, fishaman dem stap gaan dah sii go bring fish, ahn only waa go ker ahn bring tuorist. Dat definietly dah wan advantij weh dih ada ayslant dem ga uova disya archipielago, dem neva stap plant, dem neva stap minin, dem neva stap export, wii gi op eryting fih staat pen pahn turism anh govament incom. So, now San Andres sidon pahn dih fac seh iina poblik prokyorment wii ongl dipen pahn dih kolombyan mien lan prodyusars ahn somtaim dih sela dem fahn dih aylant, bot ef wih cuda embries wih lokieshan ahn agriiments weh Colombia saign, fih move prodoks around dih karibyan wih wuda gat wan secant opshan fih divelopment and exchienj. Ahn dis cuda definitely incentiviet back dih produkshan.

\section{KANCLUSHAN}

Dih Karibyan very daivers, yuh gat difarent languij, yuh gat difarent demography betwiin dih kontry dem, difarent economical activity ahn so, bot besai 
a dat karibyan kontry dem alwies deh luck how fih keep dih tai betwiin wan anada, dem staat wid dih CARIFTA ahn now dem ga dih CARICOM, ahn iivn seh dem still gat wan pail a chalenj fih get uova, dih CARICOM riily ripresent wan stronga pasability fih negochiet internashonaly. Dih poblik prokyorment deh shuo op all ova dih world laik wan riily important resuors fih dih development a dih contry dem, ahn ina dih wan dem weh jost deh divelop, dih powa weh manipuliet wid ih is wos. Dah wan reality seh crhuu dih poblik prokyorment dih govament dem kyan incriis dem economy bai dih wie dem satisfai dih necessity a dem komiunity. Dah nuh lai niida seh jost how ih kyan gih a lat a benefit ih kya ius tuh fih personal welfier a dih wan dem weh manij it, dats wai dih important fih dih govament dem divelop mekanism fih disya instrument gih dih risolt dem weh dem want ih fih gih.

Dig karybian dah no dih excepshan, fih todeh, iich wan a dih kontry dem work dih govament prokirment wih dem uon law dem, generally dih bies pahn dih bid system. Afta dih CARICOM, Dih poblik kantrak protocuol wos definietly wan a dih best impruuvment becaaz dih pritend fih help unifai dih kontry dem towards wan public kantrak system, dis gwain help bill op informieshan ahn siem taim dinamaiz dih market towards poblik adqisishan, weh dah tuu a dih fielyo weh wen aidentifai ina disya dakioment. CARICOM bies pan some kain a bradahood bitwiin dih difarent aylant dem weh shier background ahn koltyo fahn dihWest Indies. Ahn iivn seh Colombia nuh gat wan west karibyan aidentity, San Andres ahn providnes gatti, dah wan reality dih similarity wid demya rest a ayslant, ahn dat gwain be a riil advantij fih a eventual economycal rilieshan. Far instans, Colombia saign tuu agreement wid dih karybian, Bot sadly non a demya tuu instrument eva divelop moe afta dem get dih signatior.

Ina dih archipelago, Wih opshans a soplayars ina poblik prokiurment riily ridius. As dih ada karibyan ayslant dem, San Andres gat wan defichensy ina productivity; incriis dih productivity ina dih territory ridius transportieshan kaas, ahn ina dih ada ayslant dem dih rediuse tax; according to economyc stodis, dih redocshan a tax ker op dih fiiling fih work ahn siev ina dih piipl dem, ahn siem taim dat mek dih ecoomy a dih plies gruo. Dis mek wih kancluud seh dih incriis a dih productivity fih wih ayslant, weh ker tu dih ridokshan a transportieshan kaas, plos dih benefit a dih luo tax weh meki iisia fih adquaya good fah ada pliesis, since dih ayslant dah frii port, gwain definitely work pahn dih benefit a dih ayslant economy ahn dih piipl wellfier. Dih lack a kampitens ahn opshans work guens dih efishensy a dih archipelago economycal rilieshans. So, pormit wih self fih expluor adda pasabilitis kyan definitely uopn duors fi wih. Bai dih ada wie, Colombia ga wan strong reglamentieshan ina poblik kantrak, dis kya help San Andres fih bicom dih bridge ah kaaperation bitwiin dih Kontry ahn dih Karibyan. Fors, deh put dih agriiment dem weh don saign ina acshan, ahn tuu, gih San Andres, Pravidens ahn Kethlina a speshall triiment ina demya agriiment ahn dih falowing wan dem, diu tu dih 
economycal reality ah dih archipelago ahn ih cluosnis a him piipl dem tuh dih CARICOM memba dem.

\section{REFARENS DEM}

AgEnCIA EFE. "Colombia y CARICOM acuerdan estrechar lazos y hacer crecer su comercio común". Available online: https://www.efe.com/efe/america/politica/ colombia-y-caricom-acuerdan-estrechar-lazos-hacer-crecer-su-comercio-comun/20000035-4012286 [consulted 03/07/2020].

Caribbean Community (Caricom). "Member states and associate members, 2020". Available online: https://caricom.org/member-states-and-associate-members/ [consulted 03/03/2020].

Caribbean Community (Caricom). Draft Protocol on Public Procurement for the Caribbean Community. Guyana: Gerogeown, 2017.

Caribbean Community Secretariat (caricom). "History of Caribbean community", Guyana. Available online: https://caricom.org/history-of-the-caribbeancommunity/\#: :text=The\%20Caribbean\%20Community\%20and\%20Common\%20 Market\%20(CARICOM)\%20was\%20established\%20by,effect\%20on\%20August $\% 20$ $1 \% 2 \mathrm{C} \% 201973$ [consulted 03/03/2020].

Cepal. "Latin America and the Caribbean: Growth Projections for 2019 and 2020, 2019". Available.online:https://www.cepal.org/sites/default/files/pr/files/table_ press_gdp_preliminaryoverview2019-eng.pdf [consulted 03/07/2020].

Gobernación del Departamento Archipiélago de San Andrés, Providencia y SanTA Catalina. Informe de gestión 2018. Available online: https://www.sanandres. gov.co/documentos/InfGestion2018/INFORME\%20DE\%20GESTION\%202018.pdf [consulted 03/03/2020].

Interamerican Development Bank (IDB). "Caribbean region quarterly bulletin, A look at 2019 and beyond", vol. 8, n. ${ }^{\circ}$ 4, 2019. Available online: https://publications. iadb.org/publications/english/document/Caribbean_Region_Quarterly_Bulletin_Volume_8_Issue_4_December_2019_en.pdf [consulted 03/07/2020].

James, Johannie. La travesía económica del poder. Una mirada a la bistoria de San Andrés. San Andrés. Tesis de grado, Universidad Nacional de Colombia, Sede Caribe, San Andrés, 2014.

Malcolm, Christopher P. "Caribbean Integration Within the Caricom Framework: The Socio- Historical, Economyc, And Political Dynamics of a Regional Response to a Global Phenomenon". Law \& Business Review of the Americas. SMU Dedman School of Law, 2004 
Ministry of Finance \& Public Service. "Public Sector procurement policy". Available online: https://mof.gov.jm/procurement/public-sector-procurement-policy.html. [consulted 03/07/2020].

Portafolio. "Aislado San Andrés pierde 98 millones cada mes". Available online: https:// www.portafolio.co/economya/aislado-san-andres-pierde-98-000-millones-cadames-542467.

Rodríguez, Hillary. Discriminación positiva de raizales en la contratación pública de San Andrés isla. Tesis de maestría, Universidad Externado de Colombia, Bogotá, 2018.

SALAS, Jesús. Reflexiones socio-bistóricas sobre el turismo en el Caribe insular colombiano: una lectura del puerto libre. Tesis de Grado, Universidad Nacional, Bogotá.

SÁnCHEZ JabBa, ANDrés. "Violencia y narcotráfico en San Andrés", Documentos de trabajo sobre economía regional n.o 181, Banco de la República, febrero de 2013. Disponible en línea: https://www.banrep.gov.co/sites/default/files/publicaciones/archivos/ dtser_181.pdf [consultado el 1. ${ }^{\circ}$ de agosto de 2020].

Schrouder, Sandra. Public Procurement in The Caribbean: Confronting the Challenges and Opportunities. Florida: Miami Shores, Barry University, 2010.

The World Bank. "The World Bank Is Swiftly Supporting the Efforts of The Caribbean in Facing National Emergencies Caused by COVID-19". Available online: https://www.worldbank.org/en/country/caribbean/overview.

Wilkinson, Emily. "COvid-19: A Lesson in Leadership from The Caribbean". Overseas Development Institute. Available online: https://www.odi.org/blogs/16959-covid19-lesson-leadership-caribbean. 